\title{
Pricing Warrant Bonds with Credit Risk under a Jump Diffusion Process
}

\author{
Xiaonan Su $\mathbb{D}^{1,2}$ Wei Wang, ${ }^{3}$ and Wensheng Wang $\mathbb{D}^{4}$ \\ ${ }^{1}$ School of Statistics and Mathematics, Nanjing Audit University, Nanjing 200815, China \\ ${ }^{2}$ Jiangsu Key Laboratory of Financial Engineering (Nanjing Audit University), Nanjing 211815, China \\ ${ }^{3}$ Department of Financial Engineering, Ningbo University, Ningbo 315211, China \\ ${ }^{4}$ School of Economics, Hangzhou Dianzi University, Hangzhou 310018, China
}

Correspondence should be addressed to Xiaonan Su; suxiaonanecnu@163.com

Received 1 February 2018; Accepted 10 June 2018; Published 8 July 2018

Academic Editor: Paolo Renna

Copyright (c) 2018 Xiaonan Su et al. This is an open access article distributed under the Creative Commons Attribution License, which permits unrestricted use, distribution, and reproduction in any medium, provided the original work is properly cited.

\begin{abstract}
This article investigates the pricing of the warrant bonds with default risk under a jump diffusion process. We assume that the stock price follows a jump diffusion model while the interest rate and the default intensity have the feature of mean reversion. By the risk neutral pricing theorem, we obtain an explicit pricing formula of the warrant bond. Furthermore, numerical analysis is provided to illustrate the sensitivities of the proposed pricing model.
\end{abstract}

\section{Introduction}

In recent years, warrant bond is one of the major investment instruments in financial market. The warrant bonds are made to keep the features of both convertible bonds and warrants. The holder may convert the bond into a predetermined number of stock or continue to hold the bond to maturity depending on the market. Differently from the convertible bond, the essential characteristic of the warrant bond is that the bond and the option are separable. That is to say, when the bond is converted into stock, the value of the bond still exists.

The seminal work of Brennan and Schwartz [1] and Ingersoll [2] popularized the studies on pricing convertible bond. Liao and Huang [3] considered the pricing of convertible bond with credit risk under the geometric Brownian motion model. Zhou and Wang [4] assumed that the interest rate follows the geometric Brownian motion and obtained the valuation of convertible bond by the method of measure transformation. Laura and Ioannis [5] defined the firm's optimal call policy and proposed the pricing framework for convertible bond based on a structural default model. There has been a considerable interest in investigating the valuation of warrant bond since the study of Payne et al. [6]. Zhu [7] extended Payne et al. [6] to a stochastic interest rate frame and considered the pricing of warrant bond. It is well known that traditional asset price models fail to handle discrete movements (such as random environment, market trends, interest rates, business cycles, etc.). To reflect the reality, Wang and Zhao [8] used a regime switching model to describe the price dynamics of asset and investigated the pricing of warrant bond. Chen [9] assumed that the stochastic interest rate and the underlying stock follow fractional Brownian motion, respectively, and deduced the pricing formula of warrant bond. Hu et al. [10] built a structure model under portfolio constraints, discussed the pricing of warrant bond and investment portfolios under prohibition of short-selling and borrowing, and obtained an arbitrage-free price interval.

The aforementioned papers have made significant contributions to the study of pricing convertible bonds and warrant bonds. Since the 2008 financial crisis, the credit risk has been one of the most important sources of risks that should be taken into account. Bond holders also face credit risk as bonds issuer may default before the bond is delivered. Among a vast amount of literature on credit risk, two main approaches are used to model credit risk: structural model and reduced form model. The structural model is originated by Black and Scholes [11]. Furthermore, Merton [12] assumed that the default is specified as the firm's asset value reaches 
a specific threshold boundary. Major investigations about the structural model are to characterize the evolution of the firm's value and capital structure. Related papers include Merton [12], Johnson and Stulz [13], Klein and Inglis [14], Ammann [15], and Wang and Wang [16]. In contrast, the reduced form model which considers that the default is controlled by an exogenous intensity process is more flexible and tractable in the real market. Since the pioneering work by Jarrow and Turnbull [17], more advanced settings and methods have been proposed on the reduced form model, such as Jarrow and Yu [18], Su and Wang [19], Liang et al. [20], and Wang et al. [21].

This article investigates the pricing of warrant bonds with credit risk. From the characteristic of the warrant bond, we find that its value can be divided into the value of a bond and the value of a call option. In order to price the warrant bond, we should utilize the theory of option pricing. It is known that certain vital features of financial time series cannot be depicted by the classical Black-Scholes models. Therefore, Merton [12] and many scholars introduced the jump diffusion process to describe the price dynamics of assets and improved the pricing model of Black-Scholes. Comparing with these studies about warrant bonds, the differences between theirs and ours are evident. First, based on Merton [12], we assume that the stock price follows a jump diffusion model in order to capture its large or sudden changes. Second, we use a reduced form model to describe the default risk. Finally, we provide numerical experiments to illustrate the effect of some parameters on the price of the warrant bond.

The rest of the paper is organized as follows. In Section 2, we give some basic assumptions of the model. In Section 3, we derive the pricing of the warrant bonds. In Section 4, we present some numerical analysis of the result obtained.

\section{Modeling Framework}

2.1. The Underlying Market. Let $T>0$ be a finite time horizon and $\left(\Omega, \mathscr{F},\left\{\mathscr{F}_{t}\right\}_{t \geq 0}, Q\right)$ be a filtered probability space satisfying the usual conditions. Let $Q$ represent an equivalent martingale measure under which the discounted asset price processes are martingales. We assume that $W_{t}^{S}, W_{t}^{r}, W_{t}^{\lambda}$ are standard Brownian motions under $Q$, and $N_{t}$ is a Poisson process with constant arrival rate $\nu(\nu>0)$. Assume that the covariance matrix of the Brownian motions $\mathbf{W}=$ $\left(W_{t}^{S}, W_{t}^{r}, W_{t}^{\lambda}\right)$ is

$$
\left(\begin{array}{ccc}
1 & \rho_{12} & \rho_{13} \\
\rho_{21} & 1 & \rho_{23} \\
\rho_{31} & \rho_{32} & 1
\end{array}\right) t
$$

where $\rho_{i j}=\rho_{j i}$, and $-1<\rho_{i j}<1$ for $i \neq j$.

We assume that the stock price $S=\left(S_{t}\right)$ follows a jump diffusion process. The dynamic of the stock price process $S_{t}$ is specified as

$$
\frac{d S_{t}}{S_{t-}}=r_{t} d t+\sigma_{1} d W_{t}^{S}-v \beta d t+d \sum_{i=1}^{N_{t}} X_{i}
$$

where $r_{t}$ is the instantaneous interest rate and $\sigma_{1}>0$ is the volatility of $S_{t}$. If the jump happens, the jump size is controlled by independent identical distributed random variables $X_{i}\left(X_{i}>-1, i=1,2, \ldots\right)$. Here, $X_{i}>-1$ is to make sure that the stock price is nonnegative. Furthermore, we denote $f(y)$ as the probability density of $\ln \left(1+X_{i}\right)$ and $\beta=$ $\mathbb{E}_{\mathrm{Q}}\left[X_{i}\right]$, where $\mathbb{E}_{\mathrm{Q}}[\cdot]$ denotes the mathematical expectation under the probability measure $Q$. Throughout this paper, we suppose that $\left\{N_{t}\right\}_{t \in[0, T]},\left\{X_{i}\right\}_{i=1,2, \ldots}$ and $\{\mathbf{W}\}_{t \in[0, T]}$ are mutually independent.

In addition, the money market account $B=\left(B_{t}\right)$ and the market interest rate $r=\left(r_{t}\right)$ are governed by

$$
\begin{aligned}
d B_{t} & =r_{t} B_{t} d t, \\
B_{0} & =1, \\
d r_{t} & =k\left(\theta-r_{t}\right) d t+\sigma_{2} d W_{t}^{r},
\end{aligned}
$$

where $k>0, \theta>0$, and $\sigma_{2}>0$ represent the speed of reversion, the long term mean level, and the volatility of $r_{t}$, respectively.

In this article, we use the reduced form model proposed in Jarrow and Turnbull [17] to model the default risk. Let $\tau$ denote the default time of the warrant bonds issuer with default intensity process $\lambda_{t}$. We model the default intensity $\lambda_{t}$ having the feature of mean reversion

$$
d \lambda_{t}=a\left(b-\lambda_{t}\right) d t+\sigma_{3} d W_{t}^{\lambda}
$$

where $a>0, b>0$, and $\sigma_{3}>0$ represent the speed of reversion, the long term mean level, and the volatility of $\lambda_{t}$, respectively.

Furthermore, the filtration $\mathscr{F}_{t}$ is generated by $\mathscr{F}_{t}=\mathscr{F}_{t}{ }_{t} \mathrm{~V}$ $\mathscr{F}_{t}^{r} \vee \mathscr{F}_{t}^{\lambda} \vee \mathscr{H}_{t}$, where $\mathscr{F}_{t}^{S}=\sigma\left(S_{s}, s \leq t\right), \mathscr{F}_{t}^{r}=\sigma\left(r_{s}, s \leq t\right)$, $\mathscr{F}_{t}^{\lambda}=\sigma\left(\lambda_{s}, s \leq t\right)$, and $\mathscr{H}_{t}=\sigma(I(\tau \leq s), s \leq t)$. Define a new filtration $\mathscr{G}_{t}=\mathscr{F}_{T}^{S} \vee \mathscr{F}_{T}^{r} \vee \mathscr{F}_{T}^{\lambda} \vee \mathscr{H}_{t}$, and $\mathscr{G}_{0}=\mathscr{F}_{T}^{S} \vee \mathscr{F}_{T}^{r} \vee \mathscr{F}_{T}^{\lambda}$.

We adopt the assumption of Jarrow and Yu [18]; the conditional and unconditional distributions of $\tau$ are given by

$$
\begin{aligned}
Q\left(\tau>t \mid \mathscr{G}_{0}\right) & =\exp \left(-\int_{0}^{t} \lambda_{s} d s\right), \\
Q(\tau>t) & =\mathbb{E}_{Q}\left[\exp \left(-\int_{0}^{t} \lambda_{s} d s\right)\right], \quad t \in[0, T] .
\end{aligned}
$$

2.2. Warrant Bonds. A warrant bond (see Payne et al. [6]) offers the investor the option to convert it into a predetermined amount of stock or continue to hold the bond to maturity. When the bond is converted into stock, the value of the bond still exists. We assume that the holder chooses to convert the bond into stock only at expiration time $T$. Thus, the value of the warrant bond can be divided into two parts, the value of a bond and the value of a European call option. The assumption about the conversion time $t^{*}$ may be more realistic if we assume that $t^{*} \in[0, T]$. Wang and Bian [22], Yang et al. [23], and Laura and Ioannis [5] considered the pricing of convertible bonds when the holder converts the bond into stocks before maturity. The major differences between their papers and this one are the following: first, Wang and Bian [22] assumed that the stock price is driven 
by a Poisson process and the interest rate is constant. Second, in Yang et al. [23] the interest rate and default intensity were assumed to be constants. Finally, Laura and Ioannis [5] described the default risk based on a structural default model. As mentioned above, we make assumptions about the stock price, the interest rate, and the default intensity as described by (2), (3), and (4). In fact, the result may not have explicit solution for the price of the warrant bond if the conversion time is chosen at any time before $T$ under our pricing frame and we shall explore such extension in future works. Then, the cash flows of the warrant bond at $T$ can be expressed as follows:

$$
\Psi_{T}= \begin{cases}P_{b}, & S_{T}<C_{v} \\ P_{b}+\alpha \gamma\left(S_{T}-C_{v}\right), & S_{T} \geq C_{v} .\end{cases}
$$

Here, $P_{b}=D e^{i T}$ denotes the value of a bond with the coupon rate $i$, and the face value $D . C_{v}$ is the agreed conversion price and $\alpha$ is the number of warrants that a bond can receive. $\gamma$ is the exercise proportion; that is, one bond can be converted into $\gamma$ shares of stocks.

In addition to the intensity of default, another important quantity in the credit risk studies is the recovery rate. As in Jarrow and $\mathrm{Yu}$ [18], we assume that the recovery rate is a constant $\omega(0 \leq \omega<1)$. When the warrant bonds issuer defaults, the value is given by $\omega$ times the payoff of the defaultfree bond at maturity. The valuation of the warrant bond with credit risk at time $T$ is given by

$$
V(T)=I_{\{\tau>T\}} \Psi_{T}+I_{\{\tau \leq T\}} \omega \Psi_{T}
$$

\section{Pricing the Warrant Bonds with Credit Risk}

In this section we investigate the pricing of the warrant bonds with credit risk. By the risk neutral valuation formula, under the equivalent martingale measure $Q$, the valuation at time $t$ of the warrant bond is given by

$$
V(t, T)=\mathbb{E}_{\mathrm{Q}}\left[e^{-\int_{t}^{T} r_{u} d u}\left(I_{\{\tau>T\}} \Psi_{T}+I_{\{\tau \leq T\}} \omega \Psi_{T}\right) \mid \mathscr{F}_{t}\right]
$$

In terms of the default intensity, we obtain the following expression:

$$
\begin{aligned}
V(t, T)= & \omega \mathbb{E}_{Q}\left[e^{-\int_{t}^{T} r_{u} d u} \Psi_{T} \mid \mathscr{F}_{t}\right] \\
& +(1-\omega) \mathbb{E}_{\mathrm{Q}}\left[e^{-\int_{t}^{T} r_{u} d u} I_{\{\tau>T\}} \Psi_{T} \mid \mathscr{F}_{t}\right] \\
= & \omega \mathbb{E}_{Q}\left[e^{-\int_{t}^{T} r_{u} d u} \Psi_{T} \mid \mathscr{F}_{t}\right] \\
& +(1-\omega) I_{\{\tau>t\}} \mathbb{E}_{Q}\left[e^{-\int_{t}^{T}\left(r_{u}+\lambda_{u}\right) d u} \Psi_{T} \mid \mathscr{F}_{t}\right] .
\end{aligned}
$$

The details about the above equation are in Su and Wang [19].
We substitute formula (6) into (9) and obtain

$$
\begin{aligned}
& V(t, T)=\omega\left\{\mathbb{E}_{\mathrm{Q}}\left[e^{-\int_{t}^{T} r_{u} d u} P_{b} \mid \mathscr{F}_{t}\right]\right. \\
& +\mathbb{E}_{\mathrm{Q}}\left[e^{-\int_{t}^{T} r_{u} d u} \alpha \gamma S_{T} I_{\left\{S_{T} \geq C_{v}\right\}} \mid \mathscr{F}_{t}\right] \\
& \left.-\mathbb{E}_{\mathrm{Q}}\left[e^{-\int_{t}^{T} r_{u} d u} \alpha \gamma C_{v} I_{\left\{S_{T} \geq C_{v}\right\}} \mid \mathscr{F}_{t}\right]\right\}+(1-\omega) \\
& \cdot I_{\{\tau>t\}}\left\{\mathbb{E}_{\mathrm{Q}}\left[e^{-\int_{t}^{T}\left(r_{u}+\lambda_{u}\right) d u} P_{b} \mid \mathscr{F}_{t}\right]\right. \\
& +\mathbb{E}_{\mathrm{Q}}\left[e^{-\int_{t}^{T}\left(r_{u}+\lambda_{u}\right) d u} \alpha \gamma S_{T} I_{\left\{S_{T} \geq C_{v}\right\}} \mid \mathscr{F}_{t}\right] \\
& \left.-\mathbb{E}_{\mathrm{Q}}\left[e^{-\int_{t}^{T}\left(r_{u}+\lambda_{u}\right) d u} \alpha \gamma C_{v} I_{\left\{S_{T} \geq C_{v}\right\}} \mid \mathscr{F}_{t}\right]\right\} .
\end{aligned}
$$

For simplifying the notations, denote

$$
\begin{aligned}
& I_{1}=\mathbb{E}_{\mathrm{Q}}\left[e^{-\int_{t}^{T} r_{u} d u} P_{b} \mid \mathscr{F}_{t}\right] ; \\
& I_{2}=\mathbb{E}_{\mathrm{Q}}\left[e^{-\int_{t}^{T} r_{u} d u} \alpha \gamma S_{T} I_{\left\{S_{T} \geq C_{v}\right\}} \mid \mathscr{F}_{t}\right] ; \\
& I_{3}=\mathbb{E}_{\mathrm{Q}}\left[e^{-\int_{t}^{T} r_{u} d u} \alpha \gamma C_{v} I_{\left\{S_{T} \geq C_{v}\right\}} \mid \mathscr{F}_{t}\right] ; \\
& I_{4}=\mathbb{E}_{\mathrm{Q}}\left[e^{-\int_{t}^{T}\left(r_{u}+\lambda_{u}\right) d u} P_{b} \mid \mathscr{F}_{t}\right] ; \\
& I_{5}=\mathbb{E}_{\mathrm{Q}}\left[e^{-\int_{t}^{T}\left(r_{u}+\lambda_{u}\right) d u} \alpha \gamma S_{T} I_{\left\{S_{T} \geq C_{v}\right\}} \mid \mathscr{F}_{t}\right] ; \\
& I_{6}=\mathbb{E}_{\mathrm{Q}}\left[e^{-\int_{t}^{T}\left(r_{u}+\lambda_{u}\right) d u} \alpha \gamma C_{v} I_{\left\{S_{T} \geq C_{v}\right\}} \mid \mathscr{F}_{t}\right] .
\end{aligned}
$$

Then $V(t, T)$ can be rewritten as

$$
\begin{aligned}
V(t, T)= & \omega\left(I_{1}+I_{2}-I_{3}\right) \\
& +(1-\omega) I_{\{\tau>t\}}\left(I_{4}+I_{5}-I_{6}\right) .
\end{aligned}
$$

3.1. The Useful Lemmas. In the following, we calculate $I_{1}, I_{2}, I_{3}, I_{4}, I_{5}, I_{6}$, respectively. In order to use the method of measure transformation to obtain the price of the warrant bonds, we first present two lemmas to introduce two new measures $Q^{T}$ and $Q^{\lambda}$. Let $P(t, T)$ denote the price of the zero coupon bond at time $t$, with maturity $T$. From (11), we have

$$
I_{1}=P_{b} P(t, T)
$$

According to Jaimungal and Wang [24], we get the zero coupon with the affine structure as follows:

$$
P(t, T)=\exp \left(-r_{t} \sigma(t, T, k)+A(t, T)\right),
$$


where

$$
\begin{aligned}
\sigma(t, T, k)= & \frac{1-e^{-k(T-t)}}{k}, \\
A(t, T)= & \left(\theta-\frac{\sigma_{2}^{2}}{2 k^{2}}\right)(\sigma(t, T, k)-(T-t)) \\
& -\frac{\sigma_{2}^{2}}{4 k} \sigma^{2}(t, T, k) .
\end{aligned}
$$

Moreover, $P(t, T)$ satisfies

$$
d P(t, T)=r_{t} P(t, T) d t-\sigma_{2} \sigma(t, T, k) P(t, T) d W_{t}^{r} .
$$

In the presence of stochastic interest rate, we will define the forward-neutral measure $Q^{T}$ equivalent to the risk neutral measure $Q$ by Lemma 1.

Lemma 1. Let $\eta_{1 T}$ denote the Radon-Nikodým derivative

$$
\eta_{1 T}=\frac{d Q^{T}}{d Q}=\frac{P(T, T)}{P(0, T) B_{T}},
$$

and, then,

$$
\begin{aligned}
& \widetilde{W}_{t}^{r}=W_{t}^{r}+\int_{0}^{t} \sigma_{2} \sigma(u, T, k) d u, \\
& \widetilde{W}_{t}^{S}=W_{t}^{S}+\int_{0}^{t} \rho_{12} \sigma_{2} \sigma(u, T, k) d u, \\
& \widetilde{W}_{t}^{\lambda}=W_{t}^{\lambda}+\int_{0}^{t} \rho_{23} \sigma_{2} \sigma(u, T, k) d u
\end{aligned}
$$

are the standard Brownian motions under measure $Q^{T}$. The covariance matrix of $\left(\widetilde{W}_{t}^{S}, \widetilde{W}_{t}^{r}, \widetilde{W}_{t}^{\lambda}\right)$ is the same as $\left(W_{t}^{S}, W_{t}^{r}, W_{t}^{\lambda}\right)$. Moreover, the intensity of $N_{t}$ and the distribution of $X_{i}$ under $Q^{T}$ are the same as those under $Q$.

Proof. From (19) and (20), the Radon-Nikodým derivative $\eta_{1 T}$ is given by

$$
\begin{aligned}
\eta_{1 T} & =\frac{d Q^{T}}{d Q}=\exp \left\{-\int_{0}^{T} \sigma_{2} \sigma(u, T, k) d W_{u}^{r}\right. \\
& \left.-\frac{1}{2} \int_{0}^{T} \sigma_{2}^{2} \sigma^{2}(u, T, k) d u\right\} .
\end{aligned}
$$

By virtue of Girsanov's theorem, we immediately get the result of Lemma 1.

By Bayes rule, $I_{3}$ can be calculated under $Q^{T}$ :

$$
\begin{aligned}
I_{3} & =\mathbb{E}_{Q}\left[e^{-\int_{t}^{T} r_{u} d u} \alpha \gamma C_{v} I_{\left\{S_{T} \geq C_{v}\right\}} \mid \mathscr{F}_{t}\right] \\
& =\alpha \gamma C_{v} P(t, T) \mathbb{E}_{Q^{T}}\left[I_{\left\{S_{T} \geq C_{v}\right\}} \mid \mathscr{F}_{t}\right] .
\end{aligned}
$$

According to Lemma 1 and the Itô lemma, we can rewrite $S_{T}$ under $Q^{T}$ as

$$
\begin{aligned}
S_{T} & =S_{t} \exp \left\{\theta(T-t)+\left(r_{t}-\theta\right) \sigma(t, T, k)\right. \\
& -\frac{\sigma_{1}^{2}}{2}(T-t)-v \beta(T-t)+\sum_{i=N_{t}+1}^{N_{T}} \ln \left(1+X_{i}\right) \\
& +\sigma_{1}\left(\widetilde{W}_{T}^{S}-\widetilde{W}_{t}^{S}\right)+\int_{t}^{T} \sigma_{2} \sigma(u, T, k) d \widetilde{W}_{u}^{r} \\
& -\int_{t}^{T} \sigma_{2}^{2} \sigma^{2}(u, T, k) d u \\
& \left.-\int_{t}^{T} \rho_{12} \sigma_{1} \sigma_{2} \sigma(u, T, k) d u\right\} .
\end{aligned}
$$

By the law of iterated conditional expectation, we obtain that

$$
I_{3}=\alpha \gamma C_{v} P(t, T) \mathbb{E}_{Q^{T}}\left[\mathbb { E } _ { Q ^ { T } } \left[I_{\left\{S_{T} \geq C_{v}\right\}} \mid \mathscr{F}_{t}\right.\right.
$$

$$
\begin{aligned}
& \left.\left.\vee \sigma\left(\sum_{i=N_{t}+1}^{N_{T}} \ln \left(1+X_{i}\right)\right)\right] \mid \mathscr{F}_{t}\right]=\alpha \gamma C_{v} P(t, T) \\
& \cdot\left[\sum_{n=1}^{\infty} \frac{e^{-v(T-t)} v^{n}(T-t)^{n}}{n !}\right. \\
& \cdot \int_{-\infty}^{\infty} N\left(d_{3}(t, T, y)\right) f^{n}(y) d y \\
& \left.+e^{-\gamma(T-t)} N\left(d_{3}(t, T, 0)\right)\right]
\end{aligned}
$$

where $N(\cdot)$ denotes the cumulative distribution function for a standard normal random variable, $f^{n}(y)$ is the $n$-th convolution of the density function $f(y)$ of $\ln \left(1+X_{i}\right)$, and $d_{3}(t, T, y)$ is given by formula (39) in Theorem 3 . Further,

$$
\begin{aligned}
\Lambda(t, T)= & \left(\theta-\frac{\sigma_{1}^{2}}{2}-\nu \beta\right)(T-t)+\left(r_{t}-\theta\right) \sigma(t, T, k) \\
& -\int_{t}^{T} \sigma_{2}^{2} \sigma^{2}(u, T, k) d u \\
& -\int_{t}^{T} \rho_{12} \sigma_{1} \sigma_{2} \sigma(u, T, k) d u .
\end{aligned}
$$

Let

$$
X(t, T)=\mathbb{E}_{\mathrm{Q}}\left[e^{-\int_{t}^{T}\left(r_{u}+\lambda_{u}\right) d u} \mid \mathscr{F}_{t}\right]
$$

From (12), we get

$$
I_{4}=P_{b} X(t, T) .
$$


By (3) and (4), we have

$$
\begin{aligned}
\int_{t}^{T}\left(r_{u}+\lambda_{u}\right) d u= & (\theta+b)\left(r_{t}-\theta\right) \sigma(t, T, k) \\
& +\left(\lambda_{t}-b\right) \sigma(t, T, a) \\
& +\int_{t}^{T} \sigma_{2} \sigma(u, T, k) d W_{u}^{r} \\
& +\int_{t}^{T} \sigma_{3} \sigma(u, T, a) d W_{u}^{\lambda} .
\end{aligned}
$$

Direct calculation yields

$$
\begin{aligned}
& X(t, T)=\exp \left\{-(\theta+b)(T-t)-\left(r_{r}-\theta\right) \sigma(t, T, k)\right. \\
& -\left(\lambda_{t}-b\right) \sigma(t, T, a)+\frac{1}{2} \int_{t}^{T} \sigma_{2}^{2} \sigma^{2}(u, T, k) d u \\
& +\frac{1}{2} \int_{t}^{T} \sigma_{3}^{2} \sigma^{2}(u, T, k) d u \\
& \left.+\int_{t}^{T} \rho_{23} \sigma_{2} \sigma_{3} \sigma(u, T, k) \sigma(u, T, a) d u\right\} .
\end{aligned}
$$

Next, we introduce Lemma 2.

Lemma 2. Define a measure $Q^{\lambda}$ by the Radon-Nikodým derivative

$$
\eta_{2 T}=\frac{d Q^{\lambda}}{d Q}=\frac{e^{-\int_{0}^{T}\left(r_{u}+\lambda_{u}\right) d u}}{\mathbb{E}_{Q}\left[e^{-\int_{0}^{T}\left(r_{u}+\lambda_{u}\right) d u}\right]}
$$

and then

$$
\begin{aligned}
& \bar{W}_{t}^{r}=W_{t}^{r}+\int_{0}^{t} M_{1}(u) d u, \\
& \bar{W}_{t}^{\lambda}=W_{t}^{\lambda}+\int_{0}^{t} M_{2}(u) d u, \\
& \bar{W}_{t}^{S}=W_{t}^{S}+\rho_{12} \int_{0}^{t} M_{1}(u) d u+\rho_{13} \int_{0}^{t} M_{2}(u) d u
\end{aligned}
$$

are standard $Q^{\lambda}$ Brownian motions, where $M_{1}(u)=\sigma_{2} \sigma(u, T$, $k)+\rho_{23} \sigma_{3} \sigma(u, T, a)$, and $M_{2}(u)=\sigma_{3} \sigma(u, T, a)+\rho_{23} \sigma_{2} \sigma(u$, $T, k)$. The covariance matrix of $\left(\bar{W}_{t}^{S}, \bar{W}_{t}^{r}, \bar{W}_{t}^{\lambda}\right)$ is the same as $\left(W_{t}^{S}, W_{t}^{r}, W_{t}^{\lambda}\right)$. Moreover, the intensity of $N_{t}$ and the distribution of $X_{i}$ under $Q^{\lambda}$ are the same as those under $Q$.
Proof. Analogously to the proof of Lemma 1, we can get the Radon-Nikodým derivative

$$
\begin{aligned}
\eta_{2 T} & =\exp \left\{-\int_{0}^{T} \sigma_{2} \sigma(u, T, k) d W_{u}^{r}\right. \\
& -\int_{0}^{T} \sigma_{3} \sigma(u, T, a) d W_{u}^{\lambda}-\frac{1}{2} \int_{0}^{T} \sigma_{2}^{2} \sigma^{2}(u, T, k) d u \\
& -\frac{1}{2} \int_{0}^{T} \sigma_{3}^{2} \sigma^{2}(u, T, a) d u \\
& \left.-\int_{0}^{T} \rho_{23} \sigma_{2} \sigma_{3} \sigma(u, T, k) \sigma(u, T, a) d u\right\} .
\end{aligned}
$$

By virtue of Girsanov's theorem, we can complete the proof.

From Lemma 2, Itô lemma, and (2), $S_{T}$ can be written as

$$
\begin{aligned}
S_{T} & =S_{t} \exp \left\{M(t, T)+\sigma_{1}\left(\bar{W}_{T}^{S}-\bar{W}_{t}^{S}\right)\right. \\
& \left.+\int_{t}^{T} \sigma_{2} \sigma(u, T, k) d \bar{W}_{u}^{r}+\sum_{i=N_{t}+1}^{N_{T}} \ln \left(1+X_{i}\right)\right\},
\end{aligned}
$$

where

$$
\begin{aligned}
M(t, T)= & \theta(T-t)+\left(r_{t}-\theta\right) \sigma(t, T, k) \\
& -\frac{1}{2} \sigma_{1}^{2}(T-t)-\nu \beta(T-t) \\
& -\int_{t}^{T} \sigma_{2} \sigma(u, T, k) M_{1}(u) d u \\
& -\int_{t}^{T} \rho_{12} \sigma_{1} M_{1}(u) d u \\
& -\int_{t}^{T} \rho_{13} \sigma_{1} M_{2}(u) d u .
\end{aligned}
$$

Thus, by Bayes rule and the law of iterated conditional expectation, we get

$$
\begin{aligned}
I_{6} & =\alpha \gamma C_{v} X(t, T) \mathbb{E}_{Q^{\lambda}}\left[\mathbb { E } _ { Q ^ { \lambda } } \left[I_{\left\{S_{T} \geq C_{v}\right\}} \mid \mathscr{F}_{t}\right.\right. \\
& \left.\left.\vee \sigma\left(\sum_{i=N_{t}+1}^{N_{T}} \ln \left(1+X_{i}\right)\right)\right] \mid \mathscr{F}_{t}\right]=\alpha \gamma C_{v} X(t, T) \\
& \cdot\left[\sum_{n=1}^{\infty} \frac{e^{-v(T-t)} v^{n}(T-t)^{n}}{n !}\right. \\
& \cdot \int_{-\infty}^{\infty} N\left(d_{6}(t, T, y)\right) f^{n}(y) d y \\
& \left.+e^{-v(T-t)} N\left(d_{6}(t, T, 0)\right)\right],
\end{aligned}
$$

where $d_{6}(t, T, y)$ can be obtained by formula (41) in Theorem 3. 
3.2. Main Results. In the following, we give the main result in Theorem 3.

Theorem 3. The price of the warrant bond with credit risk under the jump diffusion model at time $t$ is

$$
\begin{aligned}
& V(t, T)=\omega\left[P_{b} P(t, T)-\alpha \gamma C_{v} P(t, T)\right. \\
& \cdot\left[\sum_{n=1}^{\infty} \frac{e^{-\gamma(T-t)} v^{n}(T-t)^{n}}{n !}\right. \\
& \cdot \int_{-\infty}^{\infty} N\left(d_{3}(t, T, y)\right) f^{n}(y) d y \\
& \left.+e^{-v(T-t)} N\left(d_{3}(t, T, 0)\right)\right] \\
& +\alpha \gamma S_{t}\left[e^{-\widehat{v}(T-t)} N\left(d_{2}(t, T, 0)\right)\right. \\
& +\sum_{n=1}^{\infty} \frac{e^{-\widehat{v}(T-t)} \hat{v}^{n}(T-t)^{n}}{n !} \\
& \left.\quad \int_{-\infty}^{\infty} N\left(d_{2}(t, T, y)\right) \widehat{f}^{n}(y) d y\right]+(1-\omega)
\end{aligned}
$$

$$
\begin{aligned}
& \cdot I_{\{\tau>t\}}\left[P_{b} X(t, T)+\alpha \gamma X(t, T) \tilde{Y}(t, T)\right. \\
& \cdot\left[e^{-v^{*}(T-t)} N\left(d_{5}(t, T, 0)\right)\right. \\
& +\sum_{n=1}^{\infty} \frac{e^{-v^{*}(T-t)}\left(v^{*}\right)^{n}(T-t)^{n}}{n !} \\
& \left.\cdot \int_{-\infty}^{\infty} N\left(d_{5}(t, T, y)\right) f^{* n}(y) d y\right]-\alpha \gamma C_{v} X(t, T) \\
& \cdot\left[\sum_{n=1}^{\infty} \frac{e^{-v(T-t)} v^{n}(T-t)^{n}}{n !}\right. \\
& \cdot \int_{-\infty}^{\infty} N\left(d_{6}(t, T, y)\right) f^{n}(y) d y \\
& \left.+e^{-v(T-t)} N\left(d_{6}(t, T, 0)\right)\right]
\end{aligned}
$$

where $N(\cdot)$ is the cumulative distribution function of a standard normal distribution, $\widehat{v}=\nu^{*}=\nu(\beta+1)$, and $f^{n}(y)$, $\widehat{f}^{n}(y), f^{* n}(y)$ denote the $n$-th convolution of $f(y), \widehat{f}(y)$, $f^{*}(y)$, respectively. The definition of $\Lambda(t, T), X(t, T), M(t, T)$, and $\Gamma(t, T)$ can be referred to in (26), (30), (35), and (44). Further,

$$
\begin{aligned}
& d_{2}(t, T, y)=\frac{\ln \left(S_{t} / C_{v}\right)+\Gamma(t, T)+y}{\sqrt{(1 / 2) \int_{t}^{T} \sigma_{2}^{2} \sigma^{2}(u, T, k) d u+(1 / 2) \sigma_{1}^{2}(T-t)+\int_{t}^{T} \rho_{12} \sigma_{1} \sigma_{2} \sigma(u, T, k) d u}}, \\
& d_{3}(t, T, y)=\frac{\ln \left(S_{t} / C_{v}\right)+\Lambda(t, T)+y}{\sqrt{(1 / 2) \int_{t}^{T} \sigma_{2}^{2} \sigma^{2}(u, T, k) d u+(1 / 2) \sigma_{1}^{2}(T-t)+\int_{t}^{T} \rho_{12} \sigma_{1} \sigma_{2} \sigma(u, T, k) d u}}, \\
& d_{5}(t, T, y)=\frac{\ln \left(S_{t} / C_{v}\right)+M(t, T)+\int_{t}^{T} \sigma_{2} \sigma(u, T, k) M_{3}(u) d u+\int_{t}^{T} \sigma_{1} M_{4}(u) d u+y}{\sqrt{(1 / 2) \int_{t}^{T} \sigma_{2}^{2} \sigma^{2}(u, T, k) d u+(1 / 2) \sigma_{1}^{2}(T-t)+\int_{t}^{T} \rho_{12} \sigma_{1} \sigma_{2} \sigma(u, T, k) d u}}, \\
& d_{6}(t, T, y)=\frac{\ln \left(S_{t} / C_{v}\right)+M(t, T)+y}{\sqrt{(1 / 2) \int_{t}^{T} \sigma_{2}^{2} \sigma^{2}(u, T, k) d u+(1 / 2) \sigma_{1}^{2}(T-t)+\int_{t}^{T} \rho_{12} \sigma_{1} \sigma_{2} \sigma(u, T, k) d u}} .
\end{aligned}
$$

Proof. In order to calculate $I_{2}$, we first define a measure $Q^{S}$ by the Radon-Nikodým derivative

$$
\frac{d Q^{S}}{d Q}=\frac{S_{T} / B_{T}}{\mathbb{E}_{Q}\left[S_{T} / B_{T}\right]} .
$$

By Girsanov theorem, $\widehat{W}_{t}^{S}=W_{t}^{r}-\sigma_{1} t, \widehat{W}_{t}^{r}=W_{t}^{\lambda}-\rho_{12} \sigma_{1} t$, and $\widehat{W}_{t}^{\lambda}=W_{t}^{S}-\rho_{13} \sigma_{1} t$ are standard $Q^{S}$ Brownian motions. Under $Q^{S}$, the intensity of $N_{t}$ is $\nu(\beta+1)$, and the density function of $\ln \left(1+X_{i}\right)$ is $\widehat{f}(y)=e^{y} f(y) /(1+\beta)$. According to
Bayes rules and the law of iterated conditional expectation, $I_{2}$ can be calculated under $Q^{S}$ :

$$
\begin{aligned}
I_{2} & =\alpha \gamma S_{t} \mathbb{E}_{Q^{S}}\left[I_{\left\{S_{T} \geq C_{v}\right\}} \mid \mathscr{F}_{t}\right] \\
& =\alpha \gamma S_{t}\left[\sum_{n=1}^{\infty} \frac{e^{-\widehat{v}(T-t)} \widehat{\nu}^{n}(T-t)^{n}}{n !}\right. \\
& \cdot \int_{-\infty}^{\infty} N\left(d_{2}(t, T, y)\right) \widehat{f}^{n}(y) d y \\
& \left.+e^{-\widehat{\nu}(T-t)} N\left(d_{2}(t, T, 0)\right)\right]
\end{aligned}
$$


where

$$
\begin{aligned}
d_{2}(t, T, y) & =\frac{\ln \left(S_{t} / C_{v}\right)+\Gamma(t, T)+y}{\sqrt{(1 / 2) \int_{t}^{T} \sigma_{2}^{2} \sigma^{2}(u, T, k) d u+(1 / 2) \sigma_{1}^{2}(T-t)+\int_{t}^{T} \rho_{12} \sigma_{1} \sigma_{2} \sigma(u, T, k) d u}}, \\
\Gamma(t, T) & =\left(\theta+\frac{\sigma_{1}^{2}}{2}-v \beta\right)(T-t)+\left(r_{t}-\theta\right) \sigma(t, T, k)+\int_{t}^{T} \rho_{12} \sigma_{1} \sigma_{2} \sigma(u, T, k) d u .
\end{aligned}
$$

In addition, by Lemma 2 and Bayes rules, we get

$$
I_{5}=\alpha \gamma X(t, T) \mathbb{E}_{Q^{\lambda}}\left[S_{T} I_{\left\{S_{T} \geq C_{v}\right\}} \mid \mathscr{F}_{t}\right] .
$$

For the calculation of $I_{5}$, we perform a measure change to $Q^{\lambda}$ by the Radon-Nikodým derivative

$$
\frac{d Q^{*}}{d Q^{\lambda}}=\frac{S_{T}}{\mathbb{E}_{Q^{\lambda}}\left[S_{T}\right]} .
$$

Then, a direct application of Girsanov's theorem implies that $\widehat{\bar{W}}_{t}^{r}=\bar{W}_{t}^{r}-\int_{t}^{T} M_{3}(u) d u, \widehat{\bar{W}}_{t}^{S}=\bar{W}_{t}^{\lambda}-\int_{t}^{T} M_{4}(u) d u$, and $\widehat{\bar{W}}_{t}^{\lambda}=\bar{W}_{t}^{S}-\int_{t}^{T} \rho_{23} M_{3}(u) d u-\int_{t}^{T} \rho_{23} M_{4}(u) d u$ are standard $Q^{*}$ Brownian motions, where $M_{3}(u)=\sigma_{2} \sigma(u, T, k)+\rho_{12} \sigma_{1}$, and $M_{4}(u)=\sigma_{1}+\rho_{12} \sigma_{2}(u, T, k)$. The intensity of $N_{t}$ is $\nu^{*}=\nu(\beta+1)$, and the density function of $\ln \left(1+X_{i}\right)$ is $f^{*}(y)=e^{y} f(y) /(1+$ $\beta)$. Here, we can calculate $I_{5}$ in the following way:

$$
\begin{aligned}
I_{5} & =\alpha \gamma X(t, T) \mathbb{E}_{\mathrm{Q}^{\lambda}}\left[S_{T} \mid \mathscr{F}_{t}\right] \mathbb{E}_{\mathrm{Q}^{*}}\left[I_{\left\{S_{T} \geq C_{v}\right\}} \mid \mathscr{F}_{t}\right] \\
& =\alpha \gamma X(t, T) \widetilde{Y}(t, T) \mathbb{E}_{Q^{*}}\left[I_{\left\{S_{T} \geq C_{v}\right\}} \mid \mathscr{F}_{t}\right] \\
& =\alpha \gamma X(t, T) \widetilde{Y}(t, T)\left[\sum_{n=1}^{\infty} \frac{e^{-v^{*}(T-t)}\left(v^{*}\right)^{n}(T-t)^{n}}{n !}\right. \\
& \cdot \int_{-\infty}^{\infty} N\left(d_{5}(t, T, y)\right) f^{* n}(y) d y+e^{-v^{*}}(T-t) \\
& \left.\cdot N\left(d_{5}(t, T, 0)\right)\right]
\end{aligned}
$$

where

$$
\begin{gathered}
\tilde{Y}(t, T)=\mathbb{E}_{\mathrm{Q}^{\lambda}}\left[S_{T} \mid \mathscr{F}_{t}\right]=S_{t} \exp \{M(t, T) \\
+\nu \beta(T-t)+\frac{1}{2} \int_{t}^{T} \sigma_{2}^{2} \sigma^{2}(u, T, k) d u
\end{gathered}
$$

$$
\left.+\frac{1}{2} \sigma_{1}^{2}(T-t)+\rho_{12} \int_{t}^{T} \sigma_{1} \sigma_{2} \sigma(u, T, k) d u\right\} .
$$

Combining (15), (16), (25), (28), (36), (43), and (47), we can obtain the result of the theorem.

In the following, we present a few remarks below to discuss some special results.

Remark 4. When $\omega=1$, (37) reduces to the formula for the price of the warrant bond under a jump diffusion without credit risk. This result is consistent with Wang and Zhao [8]. In this case, (37) is simplified to

$$
\begin{gathered}
V(t, T)=P_{b} P(t, T)-\alpha \gamma C_{v} P(t, T) \\
\cdot\left[\sum_{n=1}^{\infty} \frac{e^{-\gamma(T-t)} \nu^{n}(T-t)^{n}}{n !}\right. \\
\cdot \int_{-\infty}^{\infty} N\left(d_{3}(t, T, y)\right) f^{n}(y) d y \\
\left.+e^{-v(T-t)} N\left(d_{3}(t, T, 0)\right)\right] \\
+\alpha \gamma S_{t}\left[e^{-\widehat{\nu}(T-t)} N\left(d_{2}(t, T, 0)\right)\right. \\
+\sum_{n=1}^{\infty} \frac{e^{-\widehat{\nu}(T-t)} \widehat{\nu}^{n}(T-t)^{n}}{n !} \\
\left.\cdot \int_{-\infty}^{\infty} N\left(d_{2}(t, T, y)\right) \widehat{f}^{n}(y) d y\right] .
\end{gathered}
$$

Remark 5. If the stock price is modeled without compound Poisson jump, the result of (37) is given by the following formula which is similar to that of Zhu [7]:

$$
\begin{aligned}
V(t, T)= & P_{b} P(t, T)-\alpha \gamma C_{v} P(t, T) N\left(d_{3}(t, T)\right) \\
& +\alpha \gamma S_{t} N\left(d_{2}(t, T)\right),
\end{aligned}
$$

where

$$
d_{2}(t, T)=\frac{\ln \left(S_{t} / C_{v}\right)+\Gamma(t, T)}{\sqrt{(1 / 2) \int_{t}^{T} \sigma_{2}^{2} \sigma^{2}(u, T, k) d u+(1 / 2) \sigma_{1}^{2}(T-t)+\int_{t}^{T} \rho_{12} \sigma_{1} \sigma_{2} \sigma(u, T, k) d u}},
$$




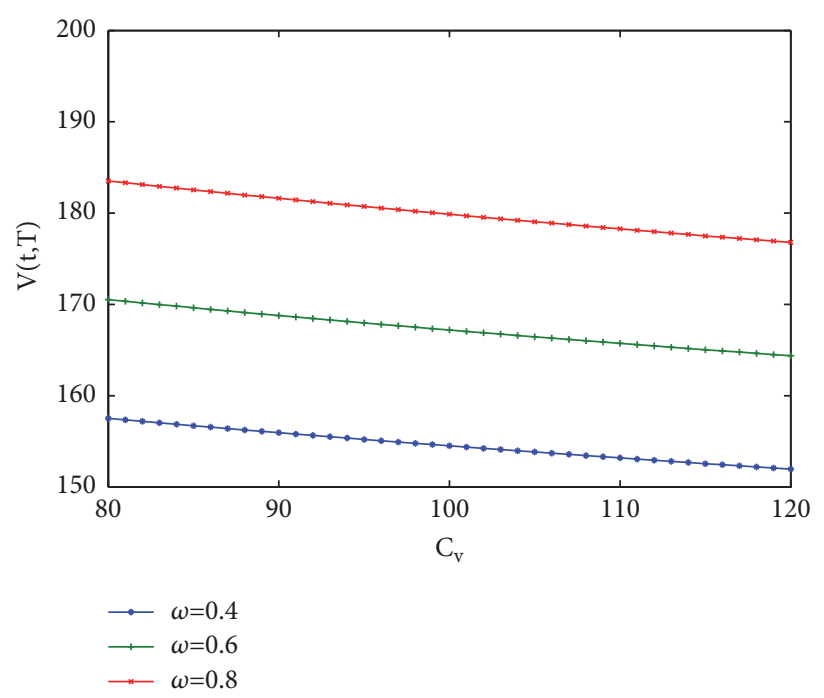

FIGURE 1: The warrant bond price with different recovery rate.

$$
\begin{aligned}
& d_{3}(t, T)=\frac{\ln \left(S_{t} / C_{v}\right)+\Lambda(t, T)}{\sqrt{(1 / 2) \int_{t}^{T} \sigma_{2}^{2} \sigma^{2}(u, T, k) d u+(1 / 2) \sigma_{1}^{2}(T-t)+\int_{t}^{T} \rho_{12} \sigma_{1} \sigma_{2} \sigma(u, T, k) d u}}, \\
& \Gamma(t, T)=\left(\theta+\frac{\sigma_{1}^{2}}{2}-v \beta\right)(T-t)+\left(r_{t}-\theta\right) \sigma(t, T, k)+\int_{t}^{T} \rho_{12} \sigma_{1} \sigma_{2} \sigma(u, T, k) d u, \\
& \Lambda(t, T)=\left(\theta-\frac{\sigma_{1}^{2}}{2}-v \beta\right)(T-t)+\left(r_{t}-\theta\right) \sigma(t, T, k)-\int_{t}^{T} \sigma_{2}^{2} \sigma^{2}(u, T, k) d u-\int_{t}^{T} \rho_{12} \sigma_{1} \sigma_{2} \sigma(u, T, k) d u .
\end{aligned}
$$

\section{Numerical Experiments}

In this section, we shall perform the numerical analysis of the results obtained in Theorem 3 . We assume that the parameters are as follows if there is no special instruction: $a=0.25, b=$ $0.1, k=0.1, \theta=0.05, \sigma_{1}=0.2, \sigma_{2}=0.2, \sigma_{3}=0.25, v=2$, $\alpha=2, \omega=0.8, i=0.05, M=100, r_{0}=0.03, S_{0}=100$, $\gamma=1, \rho_{12}=0.2, \rho_{13}=0.1, \rho_{23}=0.3, t=0$, and $T=2$. Furthermore, we assume that $\ln \left(1+X_{i}\right)$ satisfies the standard normal distribution for obtaining the numerical results of the price of the warrant bond.

In Figure 1, for each $\omega=0.4,0.6,0.8$, we consider the impact of conversion price $C_{v}$ on the warrant bond price. As mentioned above, the value of the warrant bond includes the value of a European call option, and the conversion price amounts to the exercised price of the option. So, the warrant bond price decreases as $C_{v}$ increases. It is also found in Figure 1 that the price of the warrant bond increases with the value of the recovery rate, i.e., $\omega$. In fact, the greater $\omega$ means that the holder of the warrant bond will obtain more payoff once a credit event occurs. Hence, it is not surprising that the value of the warrant bond increases as the recovery rate $\omega$ increases.
Figure 2 indicates that the initial stock price $S_{0}$ has a significant effect on the price of the warrant bond. As the values of $S_{0}$ increase, the values of the warrant bond increase as well. In fact, the greater the stock price is, the more likely the convertible bond will be converted. Hence the holder of the warrant bond can get more benefit from the higher stock price.

Figure 3 provides the impact of the exercise proportion $\gamma$ on the warrant bond. As we can see, the price of the warrant bond increases as $\gamma$ increases. In fact, the larger the exercise proportion $\gamma$, the more the stocks that can be converted into and the more the profit the holder may get which leads to the higher price of the warrant bonds.

As assumed in (4), the default intensity $\lambda$ has the property of mean reversion with the long term mean level $b$. Larger $b$ leads to the more chances of default which implies that the valuation of the warrant bond may be lower. It is shown in Figure 4 that the price of the warrant bond decreases as $b$ increases.

Finally, in Figure 5 we compare the warrant bond price with different $\theta$ which is the long term mean level of the interest rate. The value of a warrant bond includes the value of a bond and the value of an option. The higher interest rate makes the value of the bond lower but makes the value of the 


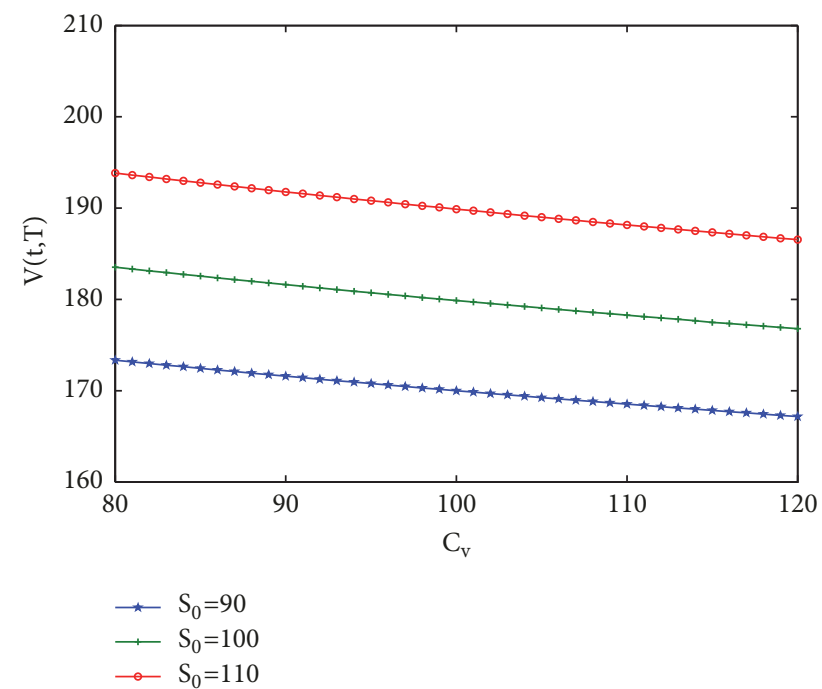

Figure 2: The warrant bond price with different $S_{0}$.

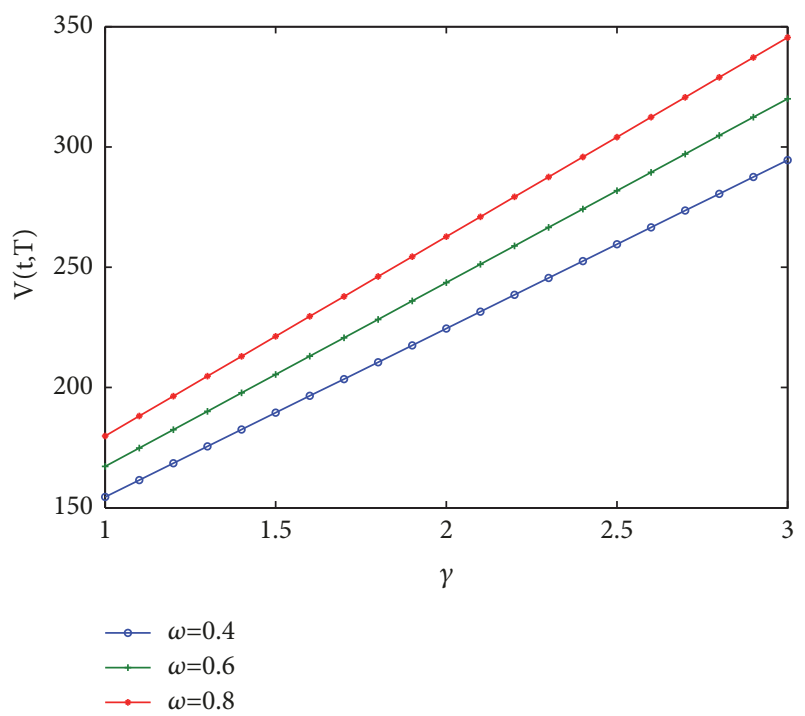

FIGURE 3: The warrant bond price with different $\gamma$.

option price higher. Combining the two facets, the higher the interest rate, the lower the price of the warrant bond.

\section{Conclusion}

The primary purpose of this paper is to value the warrant bond with credit risk under the jump diffusion model. We assume that the stock price follows a jump diffusion model while the market interest rate and the default intensity are described by mean reversion models. The technique of measure transformation is applied to provide an efficient way to evaluate the warrant bond prices. Finally, from the numerical analysis, we obtain the effects of the recovery rate $\omega$, the agreed conversion price $C_{v}$, the initial price of stock, the exercise proportion $\gamma$, and the long term mean level of interest rate and default intensity $b$ and $\theta$ on the warrant bond price.

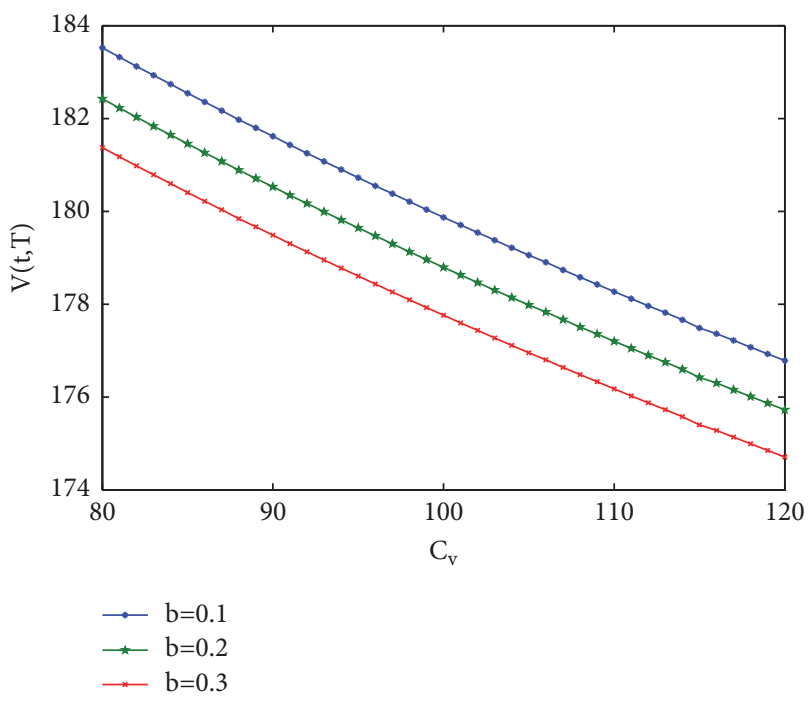

Figure 4: The warrant bond price with different $b$.

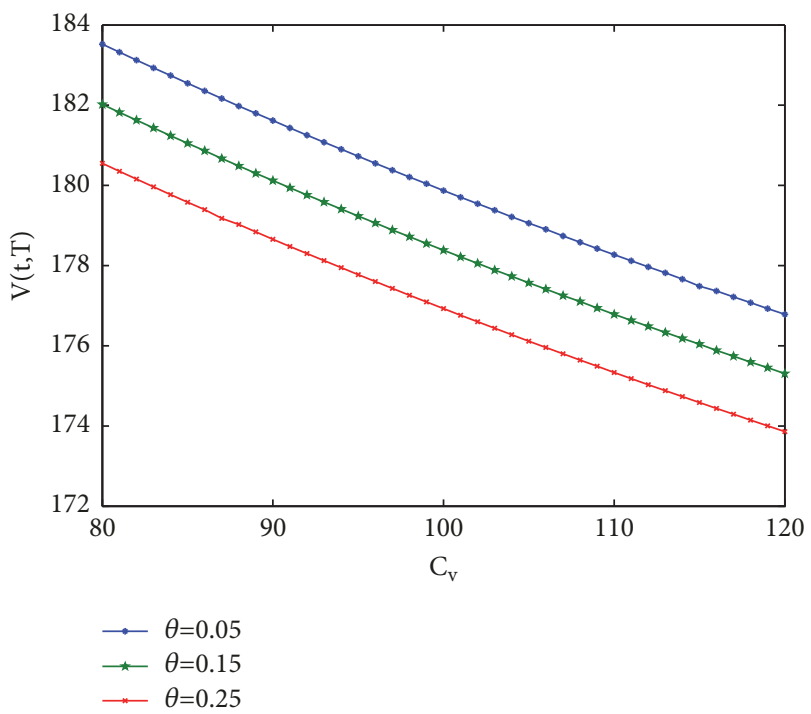

Figure 5: The warrant bond price with different $\theta$.

\section{Data Availability}

The data used to support the findings of this study are available from the corresponding author upon request.

\section{Conflicts of Interest}

There are no conflicts of interest related to this paper.

\section{Acknowledgments}

The authors gratefully acknowledge the support from the Zhejiang Provincial Natural Science Foundation of China (LY17G010003), Open Project of Jiangsu Key Laboratory of Financial Engineering (NSK2015-12), Natural Science Foundation of the Higher Education Institutions of Jiangsu 
Province (14KJB110014), and National Natural Science Foundation of China (11671115, 11526112).

\section{References}

[1] M. J. Brennan and E. S. Schwartz, "Convertible bonds: valuation and optimal strategies for call and conversion," The Journal of Finance, vol. 32, no. 5, pp. 1699-1715, 1977.

[2] J. E. Ingersoll Jr., "A contingent-claims valuation of convertible securities," Journal of Financial Economics, vol. 4, no. 3, pp. 289321, 1977.

[3] S.-L. Liao and H.-H. Huang, "Valuation and optimal strategies of convertible bonds," Journal of Futures Markets, vol. 26, no. 9, pp. 895-922, 2006.

[4] H.-1. Zhou and S.-Y. Wang, "The valuation of convertible bonds with numeraire changes," Acta Mathematicae Applicatae Sinica, vol. 26, no. 2, pp. 321-332, 2010.

[5] L. Ballotta and I. Kyriakou, "Convertible bond valuation in a jump diffusion setting with stochastic interest rates," Quantitative Finance, vol. 15, no. 1, pp. 115-129, 2015.

[6] B. C. Payne, D. O. Boudreaux, and N. C. Rumore, "Convertible bonds and bond-warrant packages: Contrasts in issuer profiles," Atlantic Economic Journal, vol. 23, no. 2, p. 152, 1995.

[7] D. Zhu, "Martingale pricing for warrant bonds under stochastic interest rate," Acta Mathematicae Applicatae Sinica, vol. 34, no. 2, pp. 265-271, 2011.

[8] W. Wang and Q. J. Zhao, "Pricing warrant bonds under a Markov-modulated jump diffusion process," Journal of East China Normal University. Natural Science Edition, vol. 6, pp. 3948, 2014.

[9] Y. Chen, "The Pricing for Warrant Bonds under Double Fractional Brownian Motion," Advances in Applied Mathematics, vol. 3, no. 4, pp. 213-221, 2014.

[10] C. Hu, Z. Cheng, J. Chen, and D. Xiong, "Pricing warrants bonds under portfolio constraints," Chinese Journal of Management, vol. 25, no. 9, pp. 11-18, 2017.

[11] F. Black and M. Scholes, "The pricing of options and corporate liabilities," Journal of Political Economy, vol. 81, no. 3, pp. 637654, 1973.

[12] R. C. Merton, "Option pricing when underlying stock returns are discontinuous," Journal of Financial Economics, vol. 3, no. 1-2, pp. 125-144, 1976.

[13] H. Johnson and R. Stulz, "The pricing of options with default ris," The Journal of Finance, vol. 42, no. 2, pp. 267-280, 1987.

[14] P. Klein and M. Inglis, "Pricing vulnerable European options when the option's payoff can increase the risk of financial distress," Journal of Banking \& Finance, vol. 25, no. 5, pp. 9931012, 2001.

[15] M. Ammann, Credit Risk Valuation: Methods, Models and Applications, Springer Finance, Springer, Berlin, Germany, 2nd edition, 2002.

[16] W. Wang and W. Wang, "Pricing vulnerable options under a Markov-modulated regime switching model," Communications in Statistics-Theory and Methods, vol. 39, no. 19, pp. 3421-3433, 2010.

[17] R. Jarrow and S. Turnbull, "Credit risk: drawing the analogy," Risk Magazine, vol. 5, no. 9, pp. 51-56, 1992.

[18] R. A. Jarrow and F. Yu, "Counterparty risk and the pricing of defaultable securities," Journal of Finance, vol. 56, no. 5, pp. 1765-1799, 2001.
[19] X. Su and W. Wang, "Pricing options with credit risk in a reduced form model," Journal of the Korean Statistical Society, vol. 41, no. 4, pp. 437-444, 2012.

[20] X. Liang, G. Wang, and H. Li, "Pricing credit default swaps with bilateral counterparty risk in a reduced form model with Markov regime switching," Applied Mathematics and Computation, vol. 230, pp. 290-302, 2014.

[21] W. Wang, Z. Jin, L. Qian, and X. Su, "Local risk minimization for vulnerable European contingent claims on nontradable assets under regime switching models," Stochastic Analysis and Applications, vol. 34, no. 4, pp. 662-678, 2016.

[22] L.-l. Wang and B.-j. Bian, "The pricing of perpetual convertible bond with credit risk," Applied Mathematics-A Journal of Chinese Universities Series B, vol. 25, no. 3, pp. 277-290, 2010.

[23] L.-H. Yang, Y.-S. Lan, and X.-B. Cao, "Pricing model of convertible bonds with default risk in a generic Levy process," System Engineering Theory and Practice, vol. 30, no. 12, pp. 21842189, 2010.

[24] S. Jaimungal and T. Wang, "Catastrophe options with stochastic interest rates and compound Poisson losses," Insurance: Mathematics \& Economics, vol. 38, no. 3, pp. 469-483, 2006. 


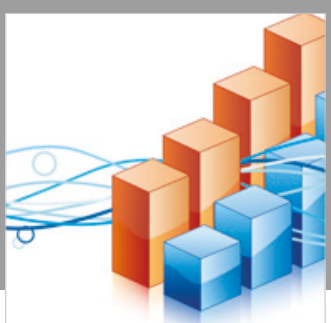

Advances in

Operations Research

\section{-n-m}
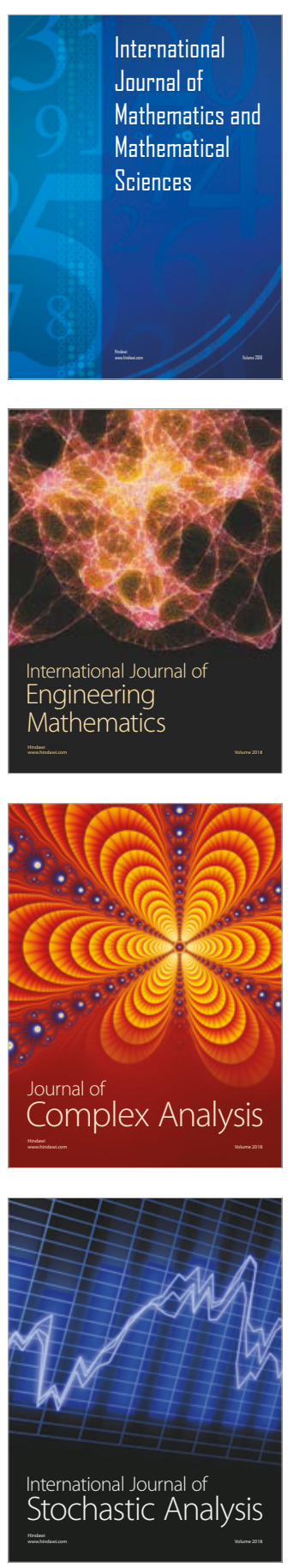
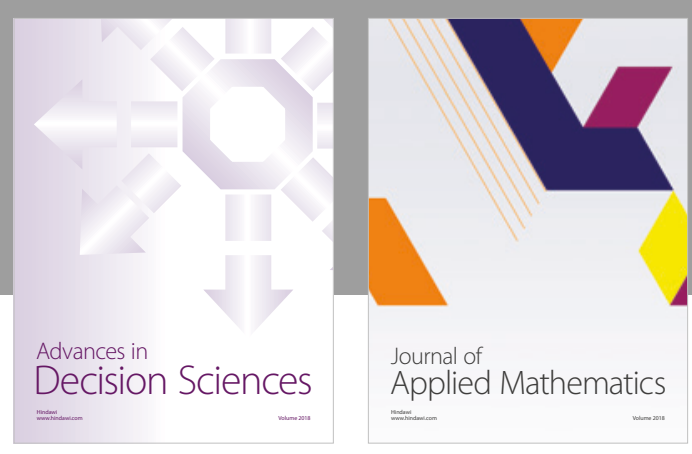

Journal of

Applied Mathematics
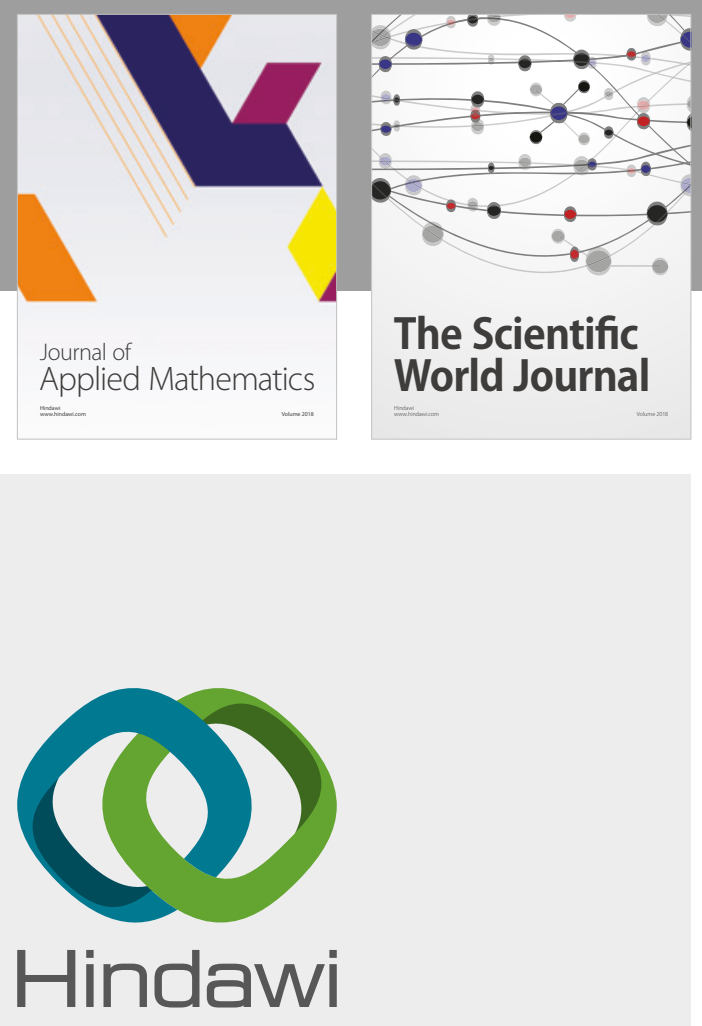

Submit your manuscripts at

www.hindawi.com

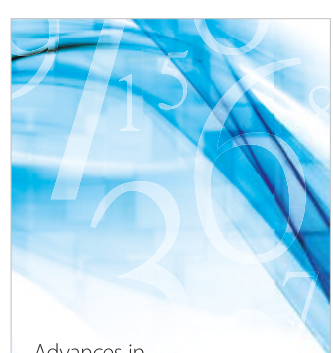

Advances in
Numerical Analysis
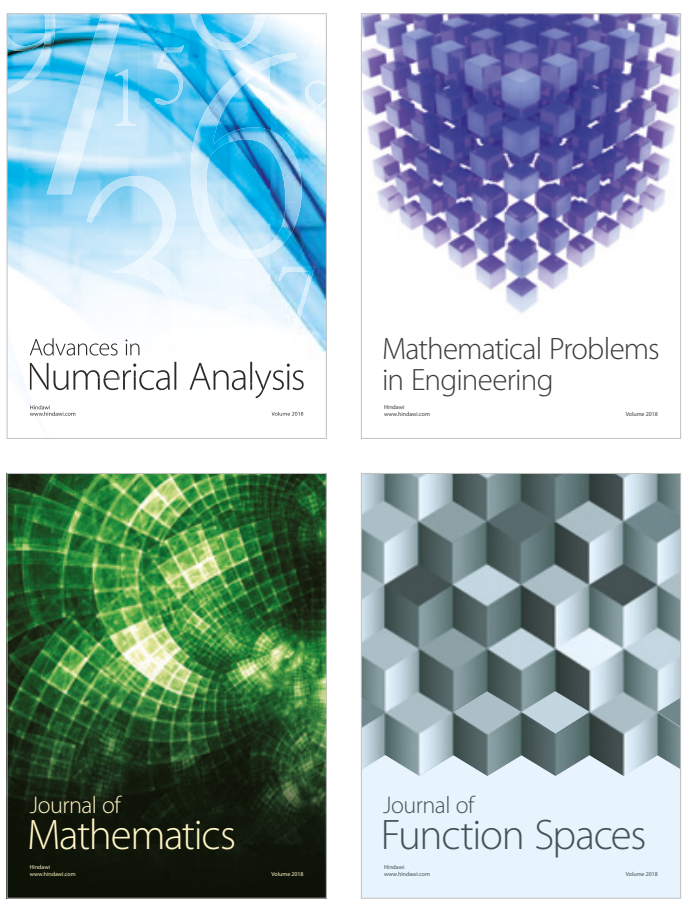

Mathematical Problems in Engineering

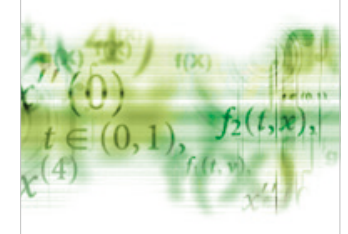

International Journal of

Differential Equations

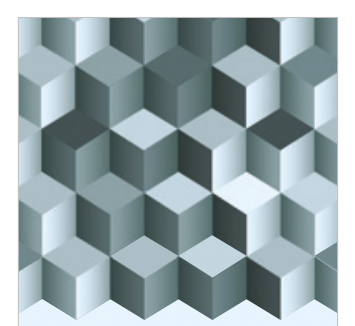

Journal of

Function Spaces

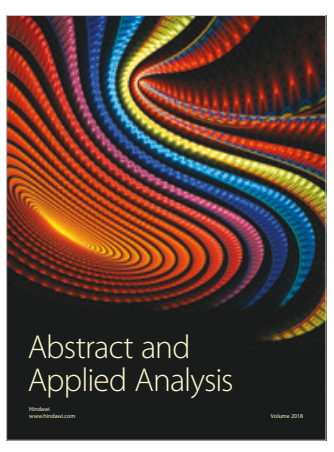

The Scientific

World Journal

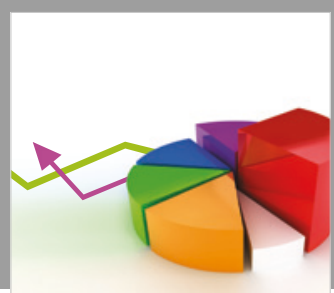

Journal of

Probability and Statistics
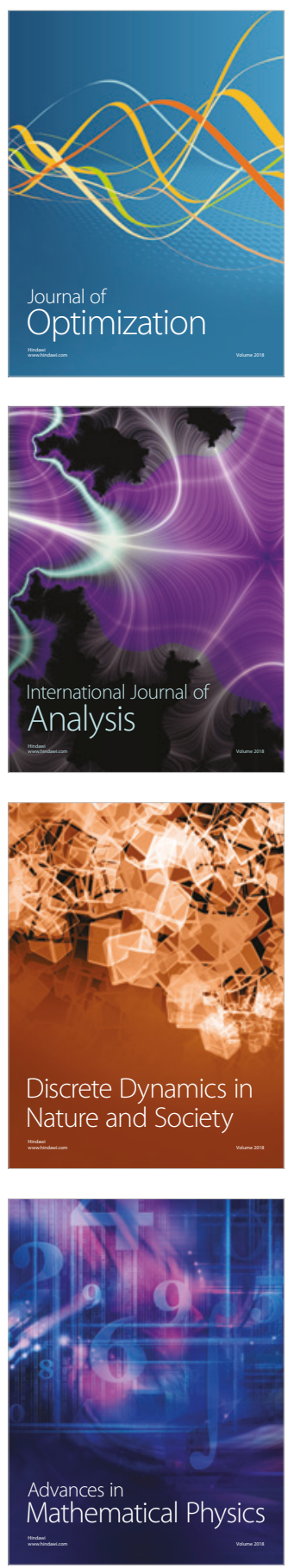